\title{
Comparative Evaluation of the Effect of Alum and Herbal Mouthrinses on Plaque Inhibition in Children: A Randomized Clinical Trial
}

\author{
BK Vanishree ${ }^{1}$, Chidanandeswara Gangadharaiah ${ }^{2}$, Shweta Kajjari ${ }^{3}$, Bharath V Sundararajan ${ }^{4}$, Nisarga Kansar ${ }^{5}$
}

\begin{abstract}
Background: Gram-positive and gram-negative bacteria that compose oral biofilms produce many metabolites that induce the formation of dental plaque. Dental plaque is the main factor for the initiation and progression of oral diseases. Plaque preventive measures like mechanical or chemical approaches can be used in combination with this.

Aims and objectives: A study was done to compare and evaluate the effects of $0.02 \mathrm{M}$ alum mouthrinse, herbal mouthrinse, and saline on plaque inhibition in children.

Materials and methods: Sixty healthy children of age-group 9-12 years were included in the study and divided into 3 groups of 20 each: group I: alum containing mouthwash $(0.02 \mathrm{M})$, group II: saline, and group III: herbal mouthwash, rinsing two times daily for 30 days. Plaque index scores were recorded from each individual on the 1st, 15th, and 30th day.

Results: Alum group (group I) showed a highly significant reduction of plaque at 1st, 15th, and 30th day when comparison to herbal group (group III) and saline group (group II).

Conclusion: Ingredients in the alum group (group I) were effective in plaque inhibition, it may serve as an alternative antimicrobial mouthwash. Further long-term study with a large population group is recommended to determine the efficacy of alum-containing mouthwash and herbal (Hi-ora) mouthwash in improving oral health status.

Keywords: Alum, Herbal, Mouthrinses, Plaque, Saline.

International Journal of Clinical Pediatric Dentistry (2021): 10.5005/jp-journals-10005-2036
\end{abstract}

\section{INTRODUCTION}

Dental plaque is a biofilm seen on oral surfaces which is considered as an etiologic factor for dental decay and gum disease. The formation of an acquired pellicle is the first stage in bacterial plaque formation. The source of energy for certain bacteria in the oral cavity is from the enamel pellicle of salivary constitutes. Dietary starch hydrolysis is by alpha-amylase, a component of acquired enamel pellicle derived from saliva provides additional glucose for metabolism by plaque microorganisms in close proximity to the tooth surface. ${ }^{1-3}$

Lactic, formic, and acetic acid are produced from fermentable carbohydrates which coincide with a $\mathrm{pH}$ drop in plaque that leads to tooth demineralization. This leads to further growth of microorganisms contributing to the cariogenicity of the dental plaque. $^{2,3}$

Plaque preventive measures like mechanical or chemical approaches can be used in combination with this. The various vehicles for delivery of chemical agents with anti-plaque are toothpastes, mouthrinses, gels, varnishes, chewing gum, spray, irrigators, etc. Antimicrobial agents may aid in protection by reducing plaque formation on the tooth surface, by inhibiting only those bacteria directly associated with oral diseases, or by inhibiting acid production or protease activity. ${ }^{4-6}$

Traditional mechanical methods have been proved inadequate for controlling plaque and caries, so endeavor on latest chemotherapeutic agents for preventing plaque-induced oral disease. Mouthrinses have been developed to deliver broadly the same functional benefits as toothpaste. Mouthwash is defined
${ }^{1}$ Department of Pediatric Dentistry, MAR Dental College, Pune, Maharashtra, India

${ }^{2}$ Department of Orthodontics, Subbaiah Institute of Dental Sciences and Hospital, Shivamogga, Karnataka, India

${ }^{3}$ Department of Pediatric and Preventive Dentistry, KLE VK Institute of Dental Sciences, Belagavi, Karnataka, India

${ }^{4}$ Department of Pediatric and Preventive Dentistry, Oxford Dental College, Bengaluru, Karnataka, India

${ }^{5}$ Department of Pediatric and Preventive Dentistry, Subbaiah Institute of Dental Sciences and Hospital, Shivamogga, Karnataka, India

Corresponding Author: BK Vanishree, Department of Pediatric Dentistry, MAR Dental College, Pune, Maharashtra, India, Phone: +91 9886100982, e-mail: vani05shree02@gmail.com

How to cite this article: Vanishree BK, Gangadharaiah C, Kajjari S, et al. Comparative Evaluation of the Effect of Alum and Herbal Mouthrinses on Plaque Inhibition in Children: A Randomized Clinical Trial. Int J Clin Pediatr Dent 2021;14(5):610-615.

Source of support: Nil

Conflict of interest: None

as a deodorizing antiseptic non-sterile aqueous solution, removes food particles, reduces bad breath, and provides a pleasant taste. Mouthwashes can reach difficult to clean areas such as interproximal surfaces and can also reduce the growth of biofilms on soft tissues. ${ }^{7-9}$ Different types of mouthwashes are used for preventing dental caries, oral malodor, gingival and periodontal diseases like chlorhexidine digluconate (antiseptic), stannous fluoride 
(anticariogenic), essential oils (antimicrobial), triclosan (antiseptic), sanguinarine (herbal extracts), cosmetic and cetylpyridinium chloride mouthwash. ${ }^{10}$

Various metal salts, polyvalent cations like tin, zinc, and aluminum (Al) as potassium sulfate salt, alum have plaque inhibitory activity so used in mouthwashes. Rinsing with a mixture of alum, salt, and vinegar for oral health was advocated by Hippocrates in ancient times. ${ }^{11}$ Alum mouthwashes used for its properties like astringent, anti-plaque, anti-gingivitis, antimicrobial, antiseptic, anti-calculus, decreasing dentinal hypersensitivity, prevention of halitosis, reduction of enamel dissolution, and symbiotic activity with fluoride. ${ }^{12-14}$ However, chemical mouthwash has many side effects, such as extrinsic staining of the teeth, temporary taste alteration, supragingival calculus formation. This has restricted its usage in children. ${ }^{15}$ Hence, attention is now turning to the use of natural antimicrobial compounds (herbal extracts). The combination of herbal extracts leads to the synergistic reduction of both dental plaque and gingival bleeding. ${ }^{9}$

Natural herbs like triphala, tulsipatra, neem, clove oil, and many more are used against various oral health problems. ${ }^{5,16} \mathrm{Hi}$-ora is a liquid that acts as an oral antiseptic, prevents gum and tooth disease, prevents bad breath and mouth ulcers, and also helps in gum tightening. It does not contain alcohol and sugar and is prepared with active ingredients like Pilu (Salvadora persica), Bibhitaka (Terminalia bellerica), Nagavalli (Piper betle), Gandhapura taila (Gaultheria fragrantissima), Ela (Elettaria cardamomum), Peppermint satva (Mentha spp), and Yavanisatva (Trachyspermum ammi). Hi-ora has an antimicrobial, anti-plaque, antiseptic, analgesic effect, reduces halitosis, inhibits the growth of periodontal and cariogenic pathogens. Herbal mouthwashes are now considered as an alternative and metallic compounds have been used in the past for their antimicrobial properties. ${ }^{17}$

However, very few studies have assessed the effect of alum mouthwash and herbal mouthwash on plaque inhibition. Hence, this study aimed to evaluate the effect of the alum mouthwash on plaque inhibition among 9-12 years old children.

\section{Materials and Methods}

Double-blind randomized three parallel groups were taken among 9-12 years old children. Consent was obtained from the local ethical committee and permission was sought from the concerned study participants. Survey design is given in Flowchart 1. The study sample consists of 9-12 years old 60 healthy children with a mean age of 10 years (Fig. 1). A total of 100 children were screened, of which 60 children were randomly selected which satisfied the inclusion and exclusion criteria.

They were divided into 3 groups, 20 in each group: group I: alum mouthwash $(0.02 \mathrm{M}) 0.885 \%$ hydrated aluminum potassium sulfate in distilled water at $\mathrm{pH}$ of 3-3.6. Group II: normal saline rinse (0.9\%). Group III: herbal (Hi-ora) mouthwash. The plaque status of the children was recorded using plaque indices. The duration of the study was 1 month; which was divided into three phases: 1st, 15 th, and 30th day.

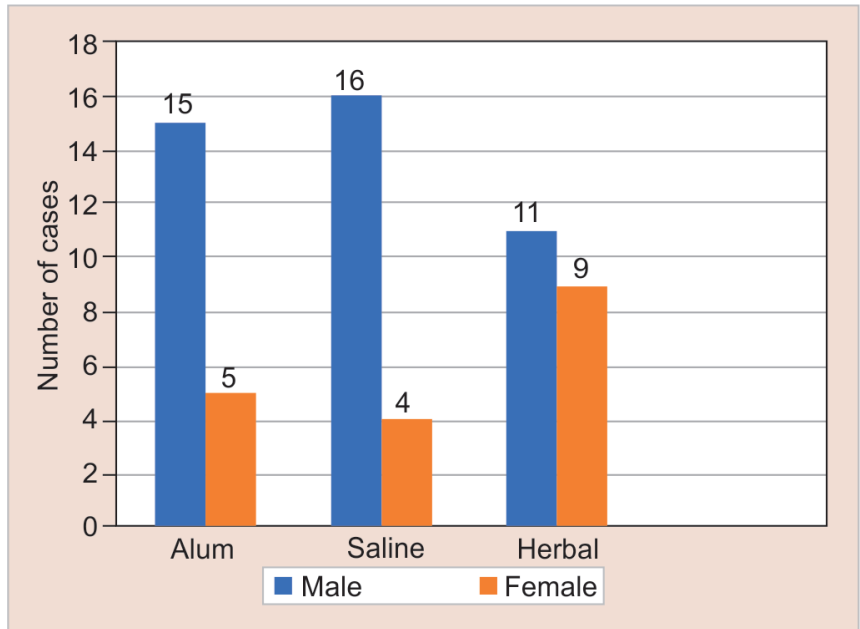

Fig. 1: Gender distribution among the study samples

Flowchart 1: Flowchart of protocol

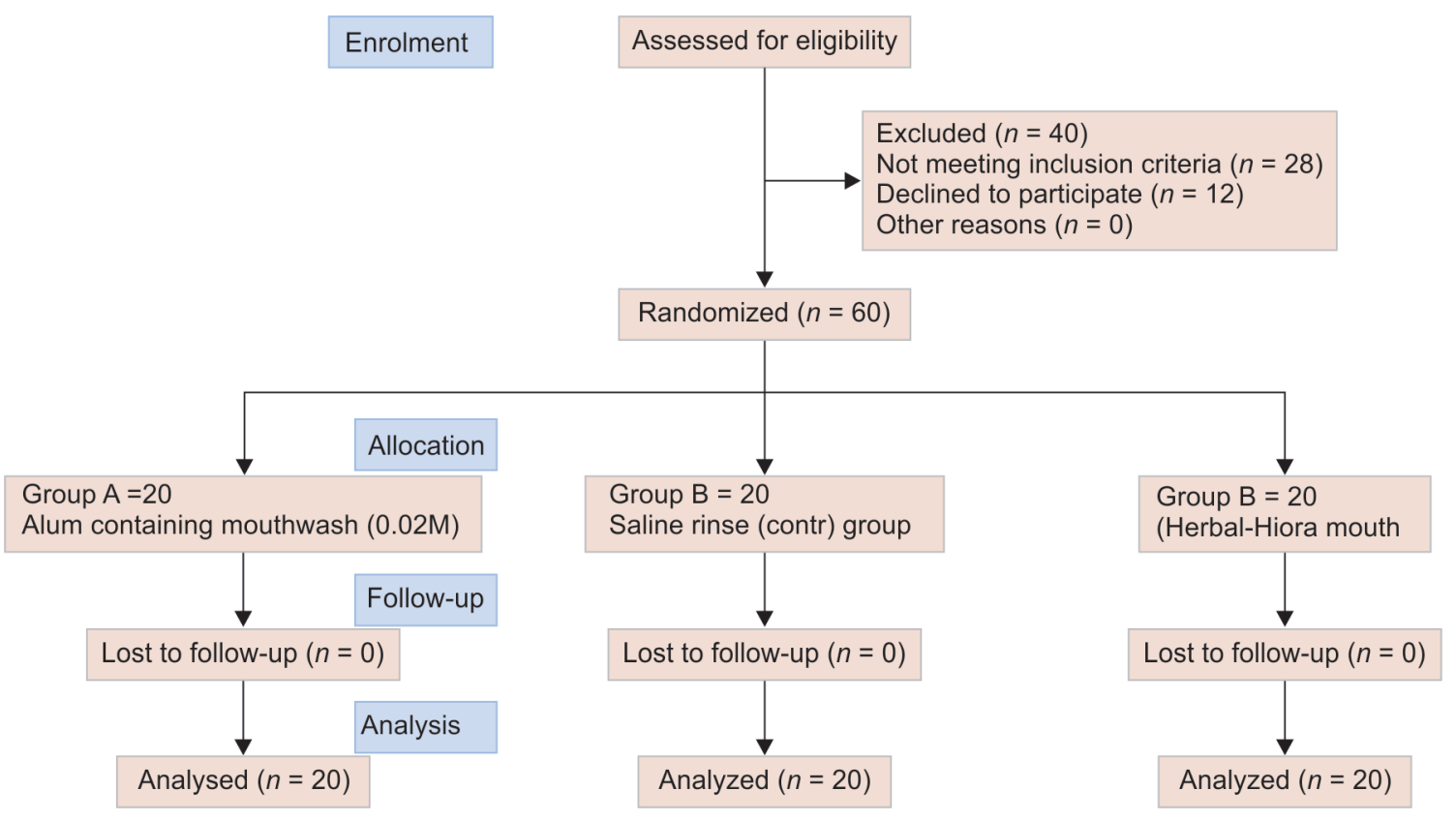




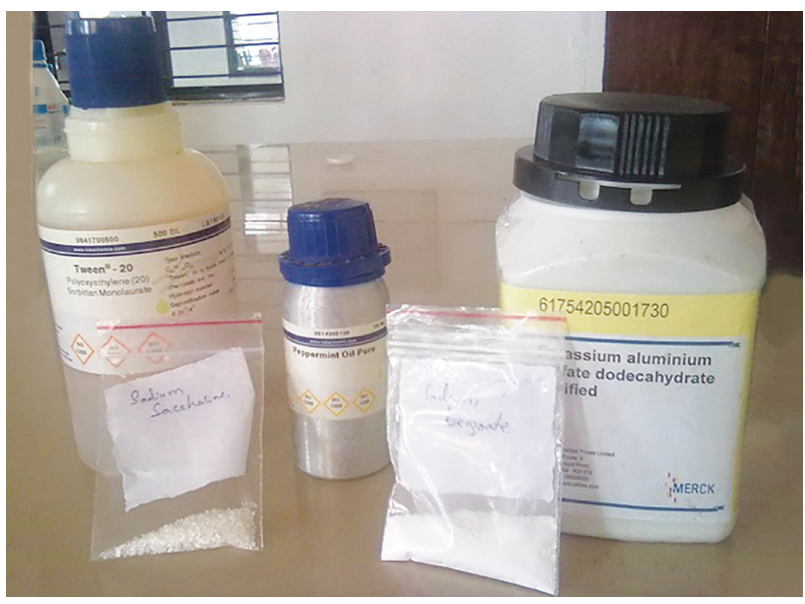

Fig. 2: Materials used for alum mouthrinse preparation

\section{Inclusion Criteria}

- 9-12 years old 60 healthy children.

- Four restored $\backslash$ decayed and $\backslash$ or missing teeth (deft/DMFT $\leq 4$ ).

\section{Exclusion Criteria}

- History of current or past 1-month antibiotic usage.

- Cellulitis, abscess, draining sinus, or other emergency dental treatment.

- Use of oral hygiene aids other than routine toothbrushing.

\section{Dental Examination Procedure}

Clinical assessments were performed by a single qualified dentist using autoclaved mouth mirror, probe, gloves, mouth mask, and portable dental operatories. Chemical sterilization (Korsolex) procedure was used to sterilize the instruments. Surface examined for a supragingival plaque of all eligible teeth are divided into mesio-facial, facial, and disto-facial, palatal or lingual surface is considered as single surface and was scored using plaque indexSillness $\mathrm{P}$ and Loe $\mathrm{H}$ in 1964.

\section{Method of Preparing 0.02 M Potash Alum Solutions ${ }^{18,19}$}

The weighed quantity of potassium aluminum sulfate is usually found in its dodecahydrate form (molecular formula) $\mathrm{KAI}\left(\mathrm{SO}_{4}\right)_{2}$ $12 \mathrm{H}_{2} \mathrm{O}$ was calculated using its molecular weight $474.39 \mathrm{wt}$ of alum $=$ mol wt $\times 0.02=9.4878 \mathrm{~g}$ was dissolved initially in $800 \mathrm{~mL}$ of distilled water, to which was added $1 \mathrm{~g}$ of sodium benzoate (as a preservative), $0.5 \mathrm{~g}$ of sodium saccharine (as a sweetening agent). In another beaker, $200 \mathrm{~mL}$ of water was taken and to it, $0.5 \mathrm{~mL}$ of Tween 20 , and $0.5 \mathrm{~mL}$ of peppermint oil was added and mixed properly and the resultant mixture was transferred to $800 \mathrm{~mL}$ alum solution and further mixed well with the help of a propeller mixture to get a clear solution. The $\mathrm{pH}$ of the solution was maintained between 3.0 and 3.5 and this can be adjusted by using sodium hydroxide (Fig. 2). The formula is as follow for every $1 \mathrm{~L}$ of solution:

Potassium aluminum sulfate: $9.4878 \mathrm{~g}$

Sodium benzoate: $1 \mathrm{~g}$

Sodium saccharine: $0.5 \mathrm{~g}$

Peppermint oil: $0.5 \mathrm{~mL}$

Tween 20: $0.5 \mathrm{~mL}$

Distilled water: $1000 \mathrm{~mL}$.

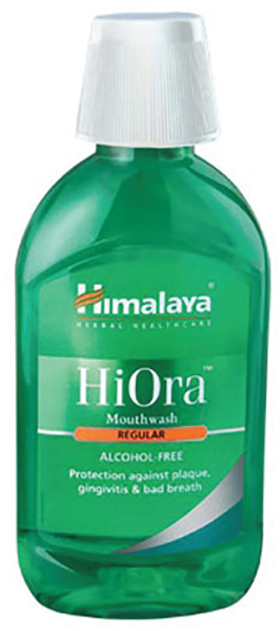

Fig. 3: Herbal-Hi-ora mouthrinses

\section{Rinsing Procedure}

Using a graduated dispensing cup, the monitor measured $10 \mathrm{~mL}$ undiluted alum and $\mathrm{Hi}$-ora mouthrinses into disposable cups (Fig. 3) and instruct the children of each group to do oral rinse by swishing $10 \mathrm{~mL}$ undiluted solution for 60 seconds once daily after meal. At the end of the investigation, beakers containing mouthrinses were coded according to the group and the data were decoded. The children were told not to eat/drink/rinse for 30 minutes after rinsing. A sufficient supply of mouthrinse, calibrated cups were given for the entire study period and told to continue normal oral hygiene procedures to children, except the use of mouthrinse. ${ }^{4}$ Following this, clinical assessments of plaque status (baseline) were done.

\section{Clinical Assessments}

Plaque index: By Sillness P and Loe H in 1964.

Surface examined for a supragingival plaque of all eligible teeth are divided into mesiofacial, facial and distofacial, palatal or lingual surface is considered as single surface and was scored using plaque index-Sillness $\mathrm{P}$ and Loe H in 1964. The plaque was scored from 0 (no plaque) to 3 (plaque covers two-thirds or more of the tooth surface) after disclosing the teeth with erythrosine $3 \%$ solution. The same procedure was repeated the 15 th and 30 th day period use of the mouthwash (Figs 4 to 6). After baseline examination, children received complete oral prophylaxis soon after receiving mouthwashes.

\section{Statistical Evaluation}

Chi-square test was used to compare the effectiveness of the alum, saline, and herbal groups at baseline, 15th day, and 30th day post rinsing. Overall group mean comparison was done by using Kruskal-Wallis test. Wilcoxon's signed ranks test for intragroup comparison and Mann-Whitney " $U$ " test for intergroup comparison of plaque status.

\section{Results}

Intergroup Comparison of Plaque Index Scores

Plaque index scores showed statistically not significant $(p=0.74)$ in any of the groups at intergroup comparison at different time intervals baseline, 15th day, and 30th day (Table 1) whereas in alum group at time-wise comparison of plaque index scores at 
baseline, 15th day, and 30th day (Table 2 ) showed highly statistically significant $(p=0.002)$.

\section{Intragroup Comparison}

Statistically no significant reduction in mean plaque scores between baseline and 15 days group I: $1.68500(p=0.653)$ vs group II: 2.20000 $(p=0.226)$ vs group III: $0.143500(p=0.837)$; between 15 days and 30 days group I: $0.321500(p=0.062)$ vs group II: $0.082500(p=1.000)$ vs group III: $0.228000(p=0.264)$.

But there was statistically high significant reduction in mean plaque scores between baseline and 30 days group I: 0.490000 ( $p=0.002)$ vs group II: $0.302500(p=0.047)$ vs group III: 0.371500 ( $p=0.019$ ) (Table 3 and Fig. 7).

\section{Discussion}

Plaque biofilm is a complex arrangement of bacteria in a self-sustaining community correlated with the initiation and progression of oral disease. Plaque preventive measures like mechanical or chemical approaches can be used in combination with this. Chemical antimicrobial agents can reach difficult to clean areas such as interproximal surfaces and can also reduce the growth of biofilms on soft tissues and as a local drug delivery agent. So, herbal and alum mouthrinse can be an alternative to other antimicrobial/antiseptic components in mouthwashes.

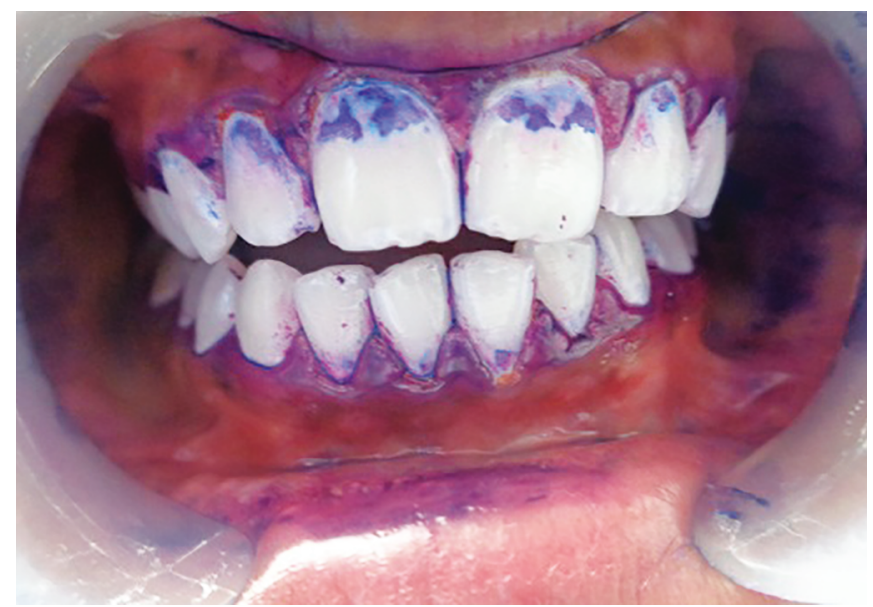

Fig. 5: Disclosing agent showing plaque status on the 15th day after using an alum mouthrinse
In the present study, a comparison with alum and herbal was done as they are the emerging components in mouthwashes and as a safe alternative to the conventional ingredients. Our study evaluated the effect of mouthwashes in plaque control in vivo. Since

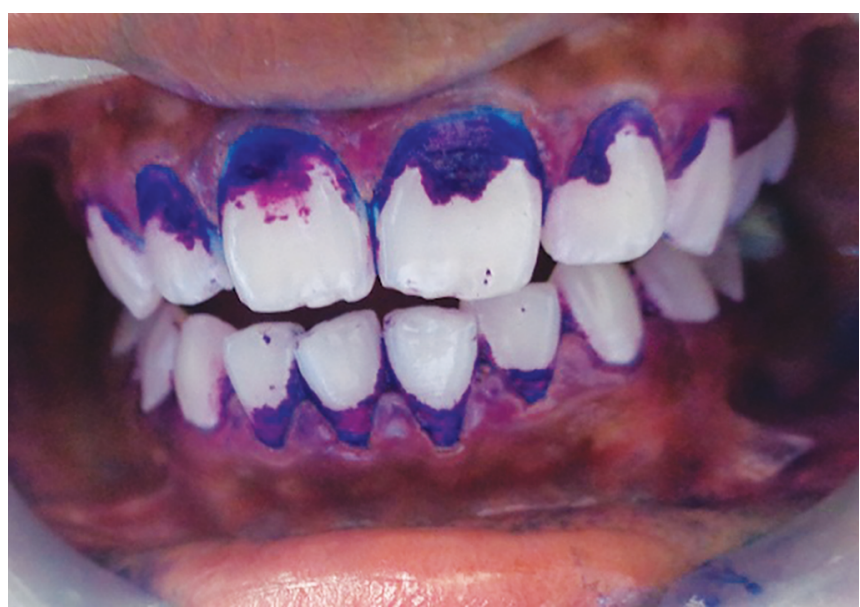

Fig. 4: Disclosing agent showing plaque status at baseline after using an alum mouthrinse

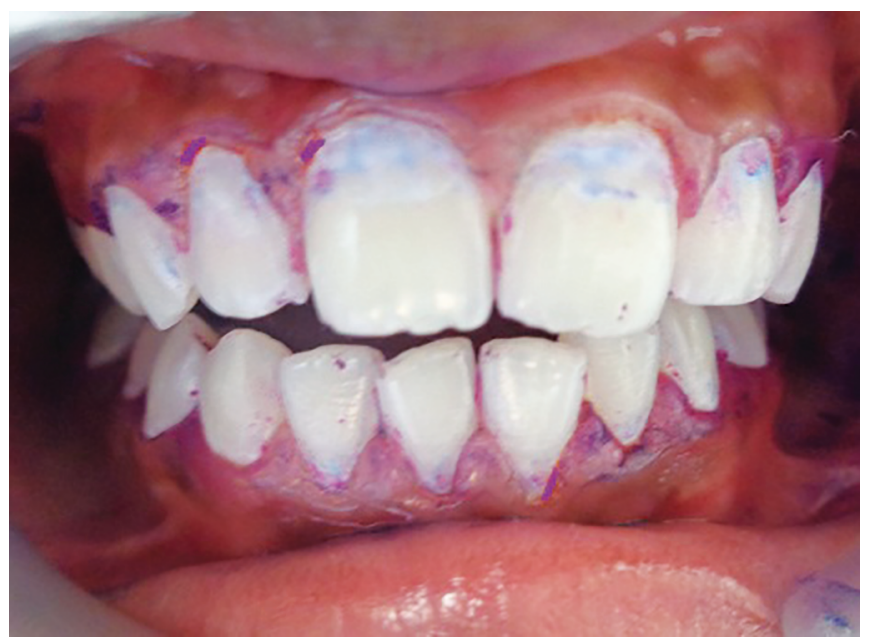

Fig. 6: Disclosing agent showing plaque status on the 30th day after using an alum mouthrinse

Table 1: Intergroup comparison of plaque index scores at baseline, 15th, and 30th days of the three groups

\begin{tabular}{llllll}
\hline Time & $N$ & Mean & Std deviation & $F$ & 0.72 \\
\hline Base alum & 20 & 1.38250 & 0.230717 & 0.34 & 0.397782 \\
Saline & 20 & 1.30750 & 0.208929 & & 0.63 \\
Herbal & 20 & 1.33750 & 0.497895 & 0.46 & 0.36 \\
15th day alum & 20 & 1.21400 & 0.368809 & & \\
Saline & 20 & 1.08750 & 1.19400 & 0.466413 & \\
Herbal & 20 & 0.89250 & 0.496640 & & \\
30th day alum & 20 & 1.00500 & 0.384537 & & \\
Saline & 20 & 0.96600 & 0.505979 & & \\
Herbal & 20 & & & \\
\hline
\end{tabular}


Table 2: Plaque index scores of the three groups at different time intervals (baseline, 15th, and 30th day)

\begin{tabular}{llllll}
\hline Groups & $N$ & Mean & Std deviation & $F$ & $0.002 \mathrm{hs}$ \\
\hline Alum base & 20 & 1.38250 & 0.230717 & 6.79 & 0.497895 \\
15th day & 20 & 1.21400 & 0.496640 & & 0.043 sig. \\
30th day & 20 & 0.89250 & 0.397782 & 3.32 & 0.02 sig. \\
Saline base & 20 & 1.30750 & 0.368809 & & \\
15th day & 20 & 1.08750 & 0.384537 & & \\
30th day & 20 & 1.00500 & 0.208929 & & \\
Herbal base & 20 & 1.33750 & 0.466413 & & \\
15th day & 20 & 0.96600 & & & \\
30th day & 20 & & & & \\
\hline
\end{tabular}

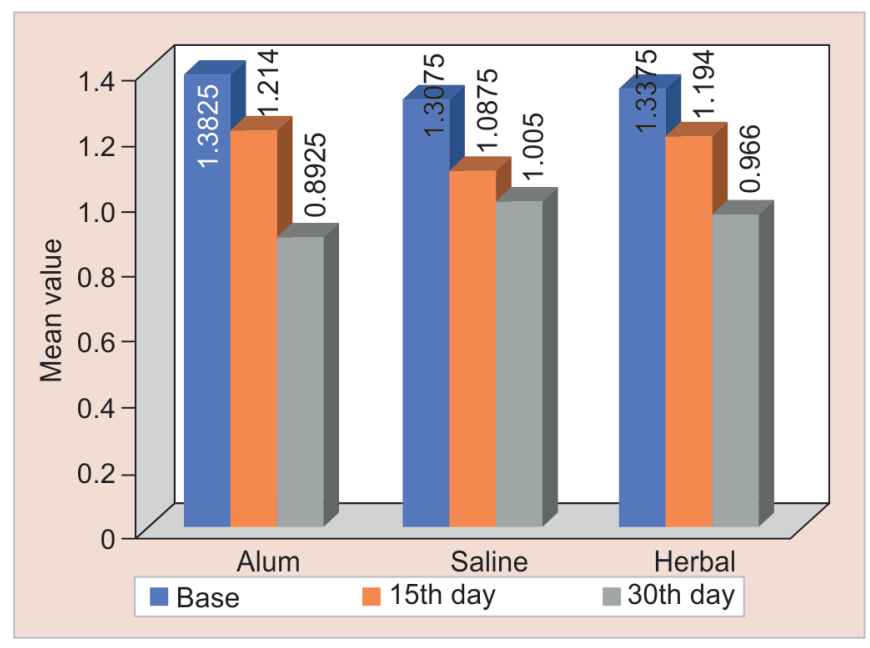

Fig. 7: Intergroup comparison of plaque index scores at baseline, 15th, and 30th days of the three groups

Table 3: Mean difference of plaque index scores at different time intervals (baseline, 15th, and 30th day)

\begin{tabular}{cll}
\hline Groups time & Mean difference & $p$ \\
\hline Alum base 15th day & 0.168500 & 0.653 \\
30th day & 0.490000 & 0.002 \\
15th day 30th day & 0.321500 & 0.062 \\
Saline base 15th day & 0.220000 & 0.226 \\
30th day & 0.302500 & 0.47 \\
15th day 30th day & 0.82500 & 1.00 \\
Herbal base 15th day & 0.143500 & 0.837 \\
30th day & 0.371500 & 0.019 \\
15th day 30th day & 0.22000 & 0.264 \\
\hline
\end{tabular}

the main effect of mouthwash is for plaque control and indices were used to evaluate the same using a disclosing agent.

Seventy percent of the population were males and $30 \%$ were females. The mean age of the population was 10.85 years in the alum group, 10.20 years in the saline group, and 9.95 years in the herbal group (Fig. 8).

\section{Effect on Plaque Status}

In the present study, we found that the mean value of plaque index scores in the alum group was found to be $1.38250 \pm 0.230717$ at baseline, $1.21400 \pm 0.497895$ on the 15 th day, and $0.89250 \pm$ 0.496640 on the 30 th day. The alum mouthrinse group showed

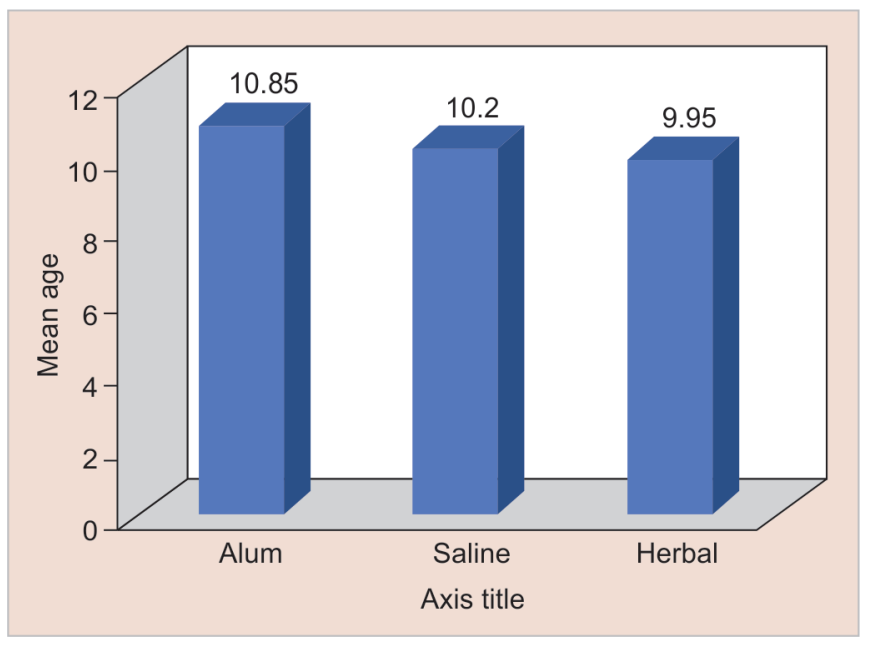

Fig. 8: Mean age distribution of samples

a very high statistically significant $(p=0.002)$ reduction in the amount of plaque on the 30th day compared to the herbal ( $p=$ $0.022)$ and saline group $(p=0.043)$. Also, a statistically significant mean difference of alum ( $p=0.002)$, herbal $(p=0.019)$, and saline $(p=0.047)$ groups between baseline and 30th day was noticed. No statistically significant ( $p=0.726$ ) between the groups. Thus, alum mouthwash emerged as the most effective adjunctive oral hygiene measure in plaque inhibition of our study.

Alum mouthwash significantly $(p<0.05)$ reduced the amount of plaque after 2 and 4 weeks, the mean value of plaque index scores in alum group was $1.58 \pm 0.11$ at 4 weeks in a study conducted by Putt et al. ${ }^{11}$ Another study by Shetty et al. showed no statistically significant $(p>0.5)$ changes between the groups (Herbal Hi-ora and Chlorhexidine mouthwash) in plaque index scores at the end of 5 days. ${ }^{20}$

Siddeshappa et al. conducted a randomized clinical trial for a period of 21 days showed a statistically significant reduction in clinical and microbiological parameters with the use of herbal, chlorine dioxide mouthwashes and concluded that herbal mouthwash was statistically efficacious in controlling plaque and gingivitis with potent antimicrobial activity. ${ }^{21}$

In contrast to our study, Al-Bayaty et al. showed a statistically highly significant difference $(p<0.001)$ in plaque index scores between the groups (herbal Salvadora persica and placebo mouthrinse). ${ }^{22}$ Another study conducted by Bajaj showed a significant difference $(p=0.001)$ in plaque scores from baseline to the end of 9 months between the groups (herbal triphala and chlorhexidine). ${ }^{23} \mathrm{~A}$ similar study conducted by Aspalli found that a 
highly significant $(p<0.0001)$ reduction in plaque scores between the groups (herbal hi-ora and non-herbal mouthwash). ${ }^{24}$ The effect of daily supervised rinsing of alum containing mouthrinse in existing plaque and gingivitis in children is determined by Putt, Kleber, Smith et al.'s study. It is known that, due to natural astringency, alum solutions can be formulated into a compatible, palatable mouthrinse use. ${ }^{25,26}$ Amount of plaque significantly $(p<$ 0.05 ) reduced by daily use of mouthrinse containing $0.02 \mathrm{M}$ alum for 30 seconds relative to the placebo. No adverse effects were observed in the oral cavity after using the alum mouthwash for 4 weeks and were accepted by the children who participated in the study. ${ }^{12}$ In addition, long-term studies need to be conducted to determine the effect of mouthwash on oral health.

\section{Conclusion}

From the present study, the following conclusions were drawn:

- The study demonstrated that alum-containing mouthwash and herbal mouthwash improve plaque inhibition.

- Alum containing mouthwash (group I) and herbal mouthwash (group III) showed a statistically significant reduction in plaque index scores at baseline, 15th day, and 30th day.

- Ingredients in the alum oral rinse were effective in reducing plaque status. Hence, it may serve as an alternative antimicrobial mouthwash.

- Further long-term study with a large population group is recommended to determine the efficacy of alum-containing mouthwashes and herbal (Hi-ora) mouthwashes in improving oral health status.

\section{References}

1. Anandan S, Nagappa. KG. Saliva-the magical fluid of the oral cavity. JIAPHD 2013;11(1):44-56.

2. Loesche WJ. Role of Streptococcus mutans in human dental decay. Microbiol Rev 1986;50(4):353-380. DOI: 10.1128/mr.50.4.353-380.1986.

3. Nyavad B, Fejerskov O. Development, structure and $\mathrm{pH}$ of dental plaque. In: Textbook of clinical cariology Thylstrup A, Fejerskov O, ed., Copenhagen: Munksgaard; 1994. pp. 89-110.

4. Nayak SS, Ankola AV. Effectiveness of mouthrinse formulated from ethanol extract of Terminalia chebula fruit on salivary Streptococcus mutans among 12-15 years old school children of Belgaum City: a randomized field trial. J Indian Soc Pedod Prev Dent 2012;30(3):231236. DOI: 10.4103/0970-4388.105016.

5. Molhotra R, Grover V, Kapoor A. Comparison of the effectiveness of a commercially available herbal mouth rinse with chlorhexidine gluconate at clinical and patient level. J Indian Soc Peridonto 2011;15(4):349-352. DOI: 10.4103/0972-124X.925672.

6. Alam A, Pravez N, Yadav S, et al. Comparative evaluation of heral mouth rinse, herbal tooth paste gel and chlorhexidine mouth rinse for cariogenic bacteria. Contin J Pharmacol Toxicol Res 2008;2: 19-26.

7. Parwani SR, Parwani RN, Chitnis PJ, et al. Comparative evaluation of anti-plaque efficacy of herbal and $0.2 \%$ chlorhexidine gluonate mouthwash in a 4-day plaque re-growth study. J Indian Soc Peridonto 2013;17(1):72-77. DOI: 10.4103/0972-124X.1074787.

8. Iqbal K, Asmat M. Role of different ingredients of tooth pastes and mouthwashes in oral health. JPDA 2011;20:163-168.

9. Shetty PR, Shetty SB, Kamat SS, et al. Comparison of the antigingivitis and antiplaque efficacy of the herboral (herbal extract) mouthwash with chlorhexidine and listerine mouthwashes: a clinical study. Pakistan Oral Dent J 2013;33(1):76-81.

10. Newman T, Klokkevold C. Carranza's clinical periodontology. 11th ed., Elsevier; 2013. pp. 1214-1219.

11. Putt MS, Kleber CJ, Smith CE. Evaluation of an alum containing mouth wash in children for plaque and gingivitis inhibition during 4 weeks of supervised use. Pediatr Dent 1996;18(2):139-144.

12. Rupesh S, Winner JJ, Nayak UA, et al. Comparative evaluation of an alum containing mouth wash and a saturated saline rinse on the salivary levels of Streptococcus mutans. J Indian Soc Pedod Prev Dent 2010;28(3):138-144.

13. Olmez $\mathrm{A}$, $\mathrm{Can} \mathrm{H}$, Ayhan $\mathrm{H}$, et al. Effect of an alum containing mouthrinse in children for plaque and salivary levels of selected oral microflora. J Clin Pediatr Dent 1998;22(4):335-340.

14. Mourughan K, Suryakanth MP. Evalution of alum containing mouth wash for inhibition of salivary S. mutans levels in children: a controlled clinical trial. J Indian Soc Pedod Prev Dent 2004;22(3):100-105.

15. Gultz J, Kaim JM, Deleo J, et al. An in vivo comparison of the antimicrobial activities of three mouth rinses. J Clin Dent 1998;9(2):43-45.

16. Nagappan N, John J. Antimicrobial efficacy of herbal and chlorhexidine mouthrinse - a systemic review. JDMS 2012;2(4):5-10.

17. Available from: http://www.en.m.wikipedia.org/wiki/Hiora.

18. Adams DS. Lab math: A hand book of measurements, calculation, and other quantitative skills for use at the bench, ch. 4. The Forsyth Institute; 2003. p. 101.

19. Adams DS. Making solutions from hydrated compound. CSH Protoc 2008. pdb.ip54. DOI: 10.1101/pdb.ip54.

20. Shetty S, Pillai S, Sridharan S, et al. Comparative efficacy of chlorhexidine and herbal mouth rinse in patients with gingival inflammation - a clinical and microbiology study. Asian J Pharmaceut Technol Innovat 2013;1:1-8.

21. Siddeshappa ST, Bhatnagar S, Yeltiwar RK, et al. Comparative evaluation of antiplaque and antigingivitis effects of an herbal and chlorine dioxide mouthwashes: a clinicomicrobiological study. Indian J Dent Res 2018;29(1):34-40. DOI: 10.4103/ijdr.IJDR_391_16.

22. Al-Bayaty FH, Al-koubaisi AH. Effect of mouth wash extracted from Salvadora persica (Miswak) on dental plaque formation: a clinical trial. J Nat Prod Med Mat 2010;4(14):1446-1454.

23. Bajaj N, Tando $S$. The effect of triphala and chlorhexidine mouthwash on dental plaque, gingival inflammation and microbial growth. Int J Ayurveda Res 2011;2(1):29-36. DOI: 10.4103/0974-7788.83188.

24. Aspalli S, Shetty VS, Devarathnamma MV, et al. Evaluation of antiplaque and antigingivitis effect of herbal mouthwash in treatment of plaque induced gingivitis; a randomized clinical trial. J Indian Soc Period 2014;18(1):48-52. DOI: 10.4103/0972-124X.128208.

25. Putt MS, Kleber CJ. Effect of $\mathrm{pH}$, concentration, and treatment time of aluminium solution on acids dissolution of enamel. J Dent Res 1986;65(11):1356-1358. DOI: 10.1177/00220345860650111601.

26. Kleber CJ, Putt MS. Investigation of the effects of aluminium mouth rinses on rat dental caries and plaque. Caries Res 1995;29(3):237-242. DOI: $10.1159 / 000262075$. 